\title{
Analysis of Risk Factors for Changes in The Doppler Echocardiographic Indexes of Patients With Gout
}

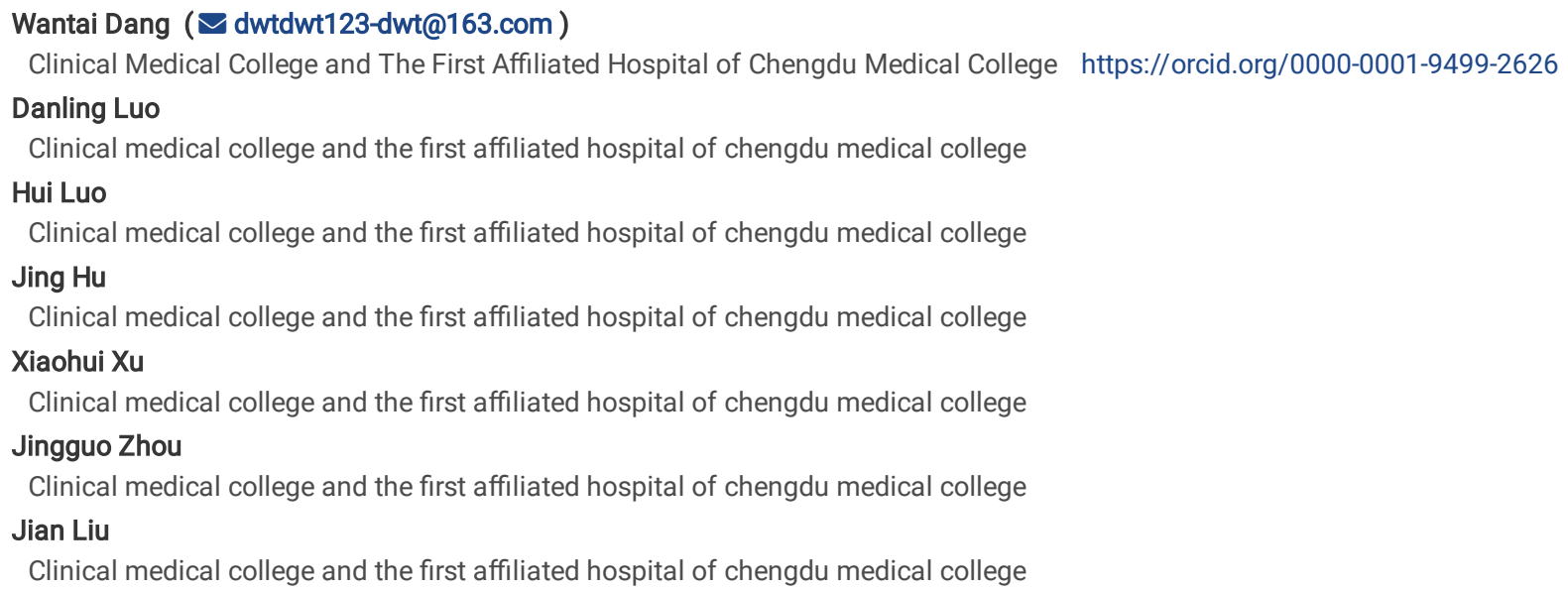




\section{Abstract}

Objective: The analysis of risk factors for changes in the Doppler echocardiographic indexes of patients with gout with different serum uric acid (SUA) levels were explored to provide clinical evidence for the early diagnosis and treatment of abnormal heart function in this patients.

Methods: We obtained information of 227 patients with primary gout (intermittent period), the left ventricular structure and functional index were measured using echocardiography and multivariate logistics regression was used to analyze the risk factors related to changes in heart function in patients with gout.

Results: Logistic regression analysis showed that the course of disease and cystatin $C$ (Cys-C) were risk factors for the increase of left atrial volume index $(\mathrm{LAVI})(\mathrm{OR}=1.14,105.47 ; 95 \% \mathrm{Cl}, 1.01-1.28,1.18-9468.03)$. Age, body mass index (BMI), course of disease, and diabetes were risk factors for the increase of the ratio of diastolic peak early transmission flow velocity to peak early diastolic mitral annulus velocity $(\mathrm{E} / \mathrm{Em})(\mathrm{OR}=1.04,1.17,1.14,4.82 ; 95 \% \mathrm{Cl}, 1.01-1.08$, 1.03-1.33, 1.02-1.27, 1.07-21.71). However, the percentage of neutrophils percentage (NE\%) and lymphocytes percentage (LY\%) might reduce the risk of left ventricular wall thickening, whereas $\mathrm{HGB}$ might reduce the risk of increasing $\mathrm{E} / \mathrm{Em}(\mathrm{OR}=0.80,0.75,0.94 ; 95 \% \mathrm{Cl}, 0.66-0.96,0.60-0.93,0.89-1.00)$.

Conclusion: Our findings suggest that BMI, course of disease, diabetes, and Cys-C are risk factors for decreased left ventricular function. Furthermore, HGB, $\mathrm{NE} \%$, and $\mathrm{LY} \%$ appear to have a weak protective effect for the remodeling of left ventricular structure and function.

\section{Introduction}

Gout is a group of clinical syndromes wherein purine metabolism disorder and decreased uric acid excretion leads to increased SUA levels and deposition of monosodium urate (MSU) crystals in tissues or organs, leading to tissue damage [1]. Studies have shown that hyperuricemia increases the risk of mortality due to coronary heart disease on the basis of traditional coronary heart disease risk factors [2, 3]. Echocardiography can be used to comprehensively and noninvasively evaluate the changes in left ventricular structure and function, and is the preferred method for clinical evaluation of cardiac function. Through echocardiographic study, Scholars in Taiwan found that the changes in left ventricular diastolic function in gout patients might be related to the inflammation mediated by monosodium urate crystals [4]. However, in patients with gout, the influence of different SUA levels on changes in cardiac function is currently unclear. This study aimed to analyze the risk factors in clinical indicators related to the changes in echocardiographic parameters in patients with gout with different SUA levels, and to provide clinical evidence for the early diagnosis and treatment of heart-related lesions in this patient group.

\section{Materials And Methods}

\subsection{Participants}

Total 227 patients with primary gout in the intermittent period were enrolled, including 215 males and 12 females. The patients were aged $16-86$ years, with an average age of $45.7 \pm 15.3$ years. The inclusion criteria were as follows: patients diagnosed with gout using the gout diagnostic criteria proposed by the American College of Rheumatology and the European League Against Rheumatism in 2015 [5]. The exclusion criteria were as follows: patients with gout secondary to malignant tumors, radiotherapy, chemotherapy, and blood system diseases; patients with previous valvular heart disease, ischemic cardiomyopathy, dilated or hypertrophic cardiomyopathy, coronary artery disease, chronic lung disease, or severe anemia; patients with arrhythmia or pacemaker implantation; patients with previous heart surgery history, or history of serious diseases.

\subsection{Ultrasonography}

Philips EPIQ 7C color Doppler ultrasound diagnostic apparatus with an S5-1 probe (frequency, 1-5MHz) was used during the experiments, and twodimensional strain analysis software was used for analysis. Routine two-dimensional echocardiography was performed on all patients included in this study. According to the recommendations of the American Echocardiography Association, the end-diastolic interventricular septum (IVS) thickness, end-diastolic left ventricular posterior wall (LVPW), and end-diastolic left ventricular end-diastolic diameter (LVEDD) were measured on the parasternal left ventricular long-axis view (see Figure 1-A), and the left ventricular ejection fraction (LVEF) was measured with the two-plane Simpson method (see Figure 1-B). The pulse Doppler sampling volume line was placed on the mitral valve orifice on the apical four-chamber view to measure the diastolic peak early transmission flow velocity (E) (Figure 1-C). The peak early diastolic mitral annulus velocity (Em) was measured through the tissue Doppler at the mitral annulus septum (see Figure 1-D) and the E/Em ratio was calculated. Moreover, two-dimensional dynamic images of three cardiac cycles of the patient's apical four-, two-, and three-chamber views, and left ventricular short-axis basal, middle, and apical sections were collected and stored. Qlab software was used to obtain the left ventricular global longitudinal strain (GLS) (Figure 1-E) and global circumferential strain (GCS) (see Figure 1-F). The apical four- and the two-chamber views were collected and LAVI was measured.

\subsection{Laboratory test}

A total of $3 \mathrm{ml}$ venous blood was collected from the patients in the morning on an empty stomach (overnight without food and drink for at least $8 \mathrm{~h}$ ), and a fully automatic blood analyzer (Japan, SYSMEX, xn-9000) was used to test the blood routine. An erythrocyte sedimentation rate analyzer (USA, VITAL diagnostics, MONITOR-100) was used to test the erythrocyte sedimentation rate. Moreover, fully automatic biochemical analyzer (Japan, Hitachi Limited, 7600) was used to measure laboratory-related indicators such as renal function, lipid metabolism, and blood sugar.

\subsection{Statistical analysis}

Quantitative data are expressed as mean \pm standard deviation or percentage, and SPSS22.0 (IBM Corporation, Armonk, NY, USA) software was used to process the data. Spearman correlation analysis was performed on laboratory indicators and other factors such as SUA and echocardiographic parameters. 
Multivariate logistic regression analysis was conducted for laboratory indicators that were correlated with echocardiographic parameters. The echocardiographic parameters were divided into two categories according to the median values. P-values $<0.05$ were considered statistically significant.

\section{Results}

\subsection{General information of the patients}

The general information of the patients is shown in Table 1.

\subsection{Correlation analysis of blood uric acid and laboratory indicators}

Spearman correlation analysis of the SUA level and other biochemical indicators in the patients with gout showed (Table 2): The SUA level was positively correlated with the course of disease, HGB, creatinine (CREA), Cys-C, total protein (TP), triglyceride (TG), total cholesterol (TC), and apolipoprotein B (ApoB), whereas it was negatively correlated with FPG $(p<0.05)$.

\subsection{Correlation analysis of echocardiographic parameters and laboratory indicators}

Spearman correlation analysis of echocardiographic parameters and laboratory indexes in patients with gout showed (Table 3): BMI, course of disease, urea (UREA), Cys-C, $\beta 2$-microglobulin ( $\beta 2-M G), T G, F P G$, glycated hemoglobin (HBA1C) and average blood glucose (AG) were positively correlated with GLS, while ApoA-1/ApoB was negatively correlated with GLS ( $p<0.05)$; GCS was positively correlated with UREA, $\beta 2-M G, C$-reactive protein (CRP) and erythrocyte sedimentation rate $(E S R)$, and negatively correlated with $L Y \%(p<0.05)$; LVEF was positively correlated with ApoA-l/ApoB, and negatively correlated with TC, low density lipoprotein cholesterol (LDL-C), ApoB and FPG ( $p<0.05)$; E/Em was positively correlated with age, BMI, course of disease, NE\%, UREA, Cys-C, $\beta 2-$ MG, lipoprotein(a) (Lp(a)), CRP and ESR, and negatively correlated with red blood cell (RBC), HGB, platelets (PLT), LY\%, TP, albumin (ALB) and albumin /globulin (A/G) ( $p<0.05)$; IVS was positively correlated with age, BMl, course of disease, white blood cell (WBC), NE\%, UREA, CREA, Cys-C, $\beta 2-M G, T G$, homocysteine (Hcy), HBA1C, AG, CRP, ESR, and negatively correlated with RBC, HGB, LY\%, TP, ALB ( $p<0.05)$; LVPW was positively correlated with age, BMI, course of disease, WBC, UREA, CREA, Cys-C, $\beta 2-M G$, TG, Hcy, HBA1C, AG, CRP and ESR, and negatively correlated with HGB, LY\%, TP, ALB, A/G and HDL-C ( $p<$ 0.05); LVEDD was positively correlated with BMI, PLT, HBA1C, AG, CRP and ESR, and negatively correlated with ALB, A/G and ApoA-I ( $<<0.05)$; LAVI was positively correlated with age, course of disease, UREA, CREA, Cys-C, $\beta 2-M G$, Lp (a), HBA1C, AG and ESR, and negatively correlated with RBC, HGB, ALB, A/G, TC and LDL-C $(p<0.05)$.

\subsection{Basic data and correlation analysis of different blood uric acid levels}

227 patients with gout were divided into 4 groups according to SUA quartile: $\leq 438 \mu \mathrm{mol} / \mathrm{L}$ (Q1 group), 439-512 $\mu \mathrm{mol} / \mathrm{L}$ (Q2 group), 513-600 $\mu \mathrm{mol} / \mathrm{L}$ (Q3 group), $>600 \mu \mathrm{mol} / \mathrm{L}$ (Q4 group). Spearman correlation analysis of echocardiographic parameters and laboratory indexes in each group showed: Q1: GLS was positively correlated with UREA ( $p<0.05)$; LVEF was positively correlated with age $(p<0.05)$; E/Em was positively correlated with age, BMI, NE\%, CRP and ESR, and negatively correlated with HGB, LY\%, TP, ALB and A/G ( $<<0.05)$; IVS was positively correlated with BMI, UREA, CREA, TG and Hcy, and negatively correlated with $\mathrm{A} / \mathrm{G}$ and HDL-C ( $<<0.05)$; LVPW was positively correlated with BMI, UREA, TG and Hcy, and negatively correlated with A/G ( $p<0.05)$; LVEDD was positively correlated with BMI, PLT and AG $(p<0.05)$; LAVI was positively correlated with age and UREA, and negatively correlated with RBC and HGB ( $<<$ 0.05). Q2: GLS was positively correlated with course of disease, HBA1C and AG, and negatively correlated with ApoA-I and ApoA-I/ApoB ( $<<0.05$ ); GCS was positively correlated with UREA and ESR $(p<0.05)$; LVEF was positively correlated with ApoA-1/ApoB, and negatively correlated with FPG ( $p<0.05)$; E/Em was positively correlated with age, course of disease, UREA, Cys-C, $\beta 2-M G$, and negatively correlated with RBC, HGB, PLT and ALB ( $p<0.05$ ); IVS was positively correlated with age, BMI, course of disease, UREA, Cys-C, $\beta 2-M G, H B A 1 C$ and AG, and negatively correlated with RBC, TP, ALB and ApoA-I ( $p<0.05$ ); LVPW was positively correlated with age, BMI, course of disease, UREA, Cys-C, $\beta 2-M G, H B A 1 C$ and AG, and negatively correlated with ALB, HDL-C and ApoA-I ( $p<0.05$ ); LVEDD was positively correlated with age, Hcy, CRP and ESR, and negatively correlated with HGB, ALB, A/G and ApoA-I ( $p<0.05)$; LAVI was positively correlated with age, Cys-C, $\beta 2-M G$, and negatively correlated with RBC, HGB, ALB, A/G, TC, LDL-C, and ApoB ( $<<0.05)$. Q3: GCS was positively correlated with UREA ( $p<0.05)$; LVEF was negatively correlated with FPG $(p<0.05)$; E/Em was positively correlated with age, course of disease, NE\%, UREA, CREA, Cys-C, $\beta 2$ $M G, H c y$ and ESR, and negatively correlated with RBC, HGB, PLT, LY\%, TP and ALB ( $<<0.05)$; IVS was positively correlated with age, course of disease, UREA, CREA, Cys-C, $\beta 2-M G$, and negatively correlated with RBC, HGB, LY\%, TP, GLB ( $p<0.05)$; LVPW was positively correlated with age, course of disease, UREA, CREA, Cys-C and $\beta 2-M G(p<0.05)$; LVEDD was positively correlated with CRP, and negatively correlated with $A / G(p<0.05)$; LAVI was positively correlated with age, course of disease, UREA and $\beta 2-M G$, and negatively correlated with HGB $(p<0.05)$. Q4: GCS was positively correlated with ESR, and negatively correlated with LY\% ( $p<0.05)$; LVEF was positively correlated with NE\%, ApoA-l/ApoB and CRP, and negatively correlated with ALB and ApoB ( $p<0.05)$; E/Em was positively correlated with age, BMI, NE\%, Cys-C , and negatively correlated with LY\% ( $<<0.05)$; IVS was positively correlated with age, BMI, course of disease, NE\%, Cys-C, $\beta 2-M G$ and ESR, and negatively correlated with RBC, HGB and LY\% ( $p<0.05)$; LVPW was positively correlated with age, BMI, course of disease, Cys-C, HBA1C and AG ( $p<0.05)$; LVEDD was positively correlated with SUA $(p<0.05)$; LAVI was positively correlated with the course of disease and negatively correlated with PLT $(p<0.05)$.

\subsection{Logistic regression analysis of echocardiographic parameters and laboratory indicators}

Logistic regression analysis showed that UREA was a risk factor for absolute reduction in GCS (OR [odds ratio] = 1.40; $95 \% \mathrm{Cl}$ [confidence interval], 1.07-1.85; $\mathrm{P}$ $=0.015)$, and that FPG was a risk factor for $L V E F$ reduction $(\mathrm{OR}=1.43 ; 95 \% \mathrm{Cl}, 1.08-1.89 ; \mathrm{p}=0.013)$. Age $(\mathrm{OR}=1.04 ; 95 \% \mathrm{Cl}, 1.01-1.08 ; \mathrm{p}=0.020)$, diabetes $(\mathrm{OR}=4.82 ; 95 \% \mathrm{Cl}, 1.07-21.71 ; \mathrm{p}=0.040), \mathrm{BMI}(\mathrm{OR}=1.17 ; 95 \% \mathrm{Cl}, 1.03-1.33 ; \mathrm{p}=0.014)$, and disease course $(\mathrm{OR}=1.14 ; 95 \% \mathrm{Cl}, 1.02-1.27 ; \mathrm{p}=0.021) \mathrm{were}$ risk factors for increased $\mathrm{E} / \mathrm{Em}$, whereas the HGB was a protective factor for increased $\mathrm{E} / \mathrm{Em}(\mathrm{OR}=0.94 ; 95 \% \mathrm{Cl}, 0.89-1.00 ; \mathrm{p}=0.032)$. Hypertension $(\mathrm{OR}=7.69$; $95 \% \mathrm{Cl}, 1.32-44.76 ; \mathrm{p}=0.023), \mathrm{BMI}(\mathrm{OR}=1.18 ; 95 \% \mathrm{Cl}, 1.02-1.37 ; \mathrm{p}=0.025)$, and course of disease $(\mathrm{OR}=1.14 ; 95 \% \mathrm{Cl}, 1.00-1.30 ; \mathrm{p}=0.046)$ were risk factors 
for thickening of IVS, whereas the NE\% (OR $=0.80 ; 95 \% \mathrm{Cl}, 0.66-0.96 ; \mathrm{p}=0.019)$ and $\mathrm{LY} \%(\mathrm{OR}=0.75 ; 95 \% \mathrm{Cl}, 0.60-0.93 ; \mathrm{p}=0.010)$ were protective factors for IVS thickening. Hypertension $(\mathrm{OR}=19.76 ; 95 \% \mathrm{Cl}, 2.68-145.55 ; \mathrm{p}=0.003)$ and $\mathrm{BMI}(\mathrm{OR}=1.26 ; 95 \% \mathrm{Cl}, 1.08-1.47 ; \mathrm{p}=0.003)$ were risk factors for $\mathrm{LVPW}$ thickening, and $\mathrm{BMI}(\mathrm{OR}=1.24 ; 95 \% \mathrm{Cl}, 1.11-1.39 ; \mathrm{p}<0.001)$ was a risk factor for increased LVPW. Course of disease $(\mathrm{OR}=1.14 ; 95 \% \mathrm{Cl}, 1.01-1.28 ; \mathrm{p}=0.02)$, Cys-C $(O R=105.47 ; 95 \% \mathrm{Cl}, 1.18-9468.03 ; \mathrm{p}=0.042)$, and ALB $(\mathrm{OR}=1.20 ; 95 \% \mathrm{Cl}, 1.01-1.43 ; \mathrm{p}=0.035)$ were risk factors for increased LAVI (Figure 2).

\section{Discussion}

Gout is a metabolic rheumatism caused by purine metabolism disorder or decreased excretion of uric acid [1]. Epidemiological studies have shown that elevated SUA can increase the risk of various diseases such as hypertension, dyslipidemia, type 2 diabetes, cardiovascular disease, and chronic kidney disease. Moreover, hypertension, diabetes, and hyperlipidemia are known risk factors for cardiovascular events [6, 7].

The results of our study showed that SUA is negatively correlated with age, which was consistent with the study results of Liang $\mathrm{J}$ et al. [8]. In addition, the FPG of patients with gout was found to be negatively correlated with SUA. Studies have shown that the blood uric acid level of healthy people increases with the increase in blood glucose concentration. Furthermore, an increasing number of studies have found that the SUA level of patients with type 2 diabetes is negatively correlated with blood glucose concentration, although the specific impact mechanism is currently unclear [9]. Cui et al. [10] found that high insulin levels played a role in the correlation between SUA and blood sugar. In our study, the disease course, renal function indexes (including CREA and Cys-C), lipid metabolism indexes (including TP, TG, TC, and ApoB), and HGB were positively correlated with SUA levels in patients with gout. Gout is an independent risk factor for impaired renal function, which is commonly evaluated by CREA and Cys-C [11]. Many studies have shown that Cys-C is an endogenous marker for evaluating early changes in renal function in patients with gout [12]. Harada et al. [13] found that high uric acid levels and Cys-C were associated with subclinical lipid abnormalities in adolescents, indicating a risk of metabolic cardiovascular disease. Studies have reported that hyperuricemia and increased lipid levels were related to fiber volume [14], which may lead to increased plaque fragility [15], and an increased risk of cardiovascular events [16].

Echocardiography is an auxiliary examination method that has developed rapidly in recent years to examine the structure and function of the heart. E/Em is a sensitive indicator reflecting left ventricular diastolic function, which increases with the decrease in left ventricular diastolic function [17]. LVEF is a commonly used indicator of left ventricular systolic function, but the decrease in LVEF generally occurs in the later stage of pathology. Many studies have reported that gout causes changes in left ventricular diastolic and systolic function [18]. Our research found that age, BMI, disease course, renal function, lipid metabolism, blood glucose, and inflammation indicators were related to left ventricular wall thickness and left ventricular diastolic function. After grouping by SUA level, regardless of SUA level, left ventricular structure and diastolic function were related to most of the above parameters, especially age, BMI, course of disease, renal function, and lipid metabolism indicators, whereas ESR was only positively correlated with IVS in the Q4 group. Moreover, in the Q4 group, LVEDD was positively correlated with SUA level, suggesting that high uric acid levels and inflammation in patients with gout aggravate left ventricular remodeling. In our study, the main manifestations of LVEF were related to lipid metabolism, and no significant changes were observed after the SUA levels were grouped. Although GLS and GCS were related to most of the patients' general conditions and laboratory-related indicators observed in our study, after logistics regression analysis, we found that only UREA was an independent risk factor for the absolute reduction of GCS. Many studies have shown that high urea levels are associated with adverse cardiovascular outcomes. Increased blood urea levels can predict renal hypoperfusion, and this renal hemodynamic status may be related to decreased cardiac output [19]. Our study showed that UREA might be a good indicator of changes in myocardial subclinical systolic function in patients with gout. This may be related to a complex neurohormonal mechanism that is activated to stimulate the release of vasopressin and activate the renal sympathetic nervous system [20].

Many studies have confirmed that patients with gout have cardiac structure and diastolic dysfunction. Our results demonstrated that BMI, disease course, and hypertension were risk factors for left ventricular wall thickening; age, BMI, disease course, and diabetes were risk factors for E/Em; and FPG was a risk factor for LVEF reduction. The exact mechanism of left ventricular diastolic dysfunction caused by gout remains unclear [21, 22]. However, it is clear that hypertension secondary to gout can aggravate the structural changes of the left ventricle and increase the risk of left ventricular hypertrophy. . Studies have suggested that patients with type 2 diabetes have abnormal cardiac structure and function [23, 24]. Indeed, a previous study reported a decrease in the Em of the mitral valve annulus in diabetic patients [25], which was consistent with our findings that diabetes was a risk factor for increased E/Em. Poor blood sugar control in diabetic patients may lead to increased left ventricular mass through myocardial cell hypertrophy, collagen deposition and cross-linking, and increased myocardial oxidative stress, which may lead to left ventricular diastolic dysfunction [26]. Previous studies used the Framingham risk score to evaluate the cardiovascular risk of obese and non-obese patients with gout. Although the results showed no significant difference between the systolic and diastolic functions of the heart, the heart morphological changes in obese patients were more pronounced [2]; this is in accordance with our findings that IVS and LVPW thickening were consistent with the risk factors of LVEDD expansion. Our results also showed that BMI was a risk factor for increased E/Em, indicating that obese patients with gout had a higher risk of decreased left ventricular diastolic function.

Left atrium remodeling has important value in predicting the risk of cardiovascular events and mortality, and left atrium enlargement plays an important role in the development of heart failure [27]. LAVI is a powerful indicator of diastolic dysfunction and is not affected by left ventricular load conditions [28]. Our findings indicated that LAVI, E/Em, and IVS increased with the development of gout, indicating that gout affected left ventricular diastolic function during the development of the disease. In addition, we found that Cys-C and ALB were independent risk factors for the increase in LAVI. A previous meta-analysis showed that there was a significant association between high levels of Cys-C and the risk of cardiovascular events in patients with normal renal function or early-stage renal dysfunction [29]. The mechanism may be related to the process of atherosclerosis [30]. Some researchers believe that the production of lysosomal cathepsin contributes to the degradation of atherosclerotic plaques. Because Cys-C can inhibit lysosomal cathepsin, increased levels of Cys- $C$ may lead to the non-degradation of atherosclerotic plaques, resulting in impaired heart function [31].

It is worth noting that our results showed that high levels of $L Y \%$ and NE\% reduced the risk of IVS thickening, suggesting that LY\% and NE\% might reduce the risk of left ventricular remodeling in patients with gout. Other studies have shown that the ratio of NE to LY (NLR) is higher than normal in patients with

Page $4 / 10$ 
coronary heart disease. Given that the NLR is an indicator of inflammation, it is believed that it could be used as an independent predictor of coronary artery disease [32,33]. Although the mechanism underlying how LY\% and NE\% affect cardiovascular changes in the context of gout is unclear, our findings suggest that $\mathrm{LY} \%$ and $\mathrm{NE} \%$ can be used to predict the possible risk of IVS thickening during clinical diagnosis and treatment. Furthermore, we found that elevated HGB was a protective factor for increased E/Em, suggesting that HGB inhibits the reduction of left ventricular diastolic function in patients with gout; this is in accordance with the findings of Burns et al. (34), who proved that HGB was negatively associated with left ventricular diastolic function.

This study explored the changes in the SUA levels, common complications, and cardiac structure and function in patients with gout, and analyzed the correlation of these indicators. We further explored the risk factors and mechanisms of gout associated with cardiac dysfunction, and provided a theoretical basis for the early diagnosis and treatment of heart disease. However, our study has some limitations that warrant discussion. First, due to the limitation of the sample size, future studies including a larger sample size should be performed to validate our findings. In addition, most of our research subjects were male patients, and sex may interfere with research factors.

\section{Conclusion}

In conclusion, our research found that patients with gout had an increased risk of abnormal heart structure and function when they were combined with obesity, hypertension, diabetes, metabolic syndrome, and other diseases. Moreover, changes in renal function had a significant impact on heart function in patients with gout. In particular, elevated Cys-C increased the risk of left ventricular diastolic dysfunction. In patients with blood uric acid levels $>438$ umol/I, Cys- $C$ was related to changes in heart function. These findings suggest that reducing uric acid while controlling Cys- $\mathrm{C}$ may be beneficial in reducing heart dysfunction in patients with gout. In our study, blood lipids were related to changes in heart function in patients with different blood uric acid levels. Suggesting that control of uric acid and lipid-lowering therapy may play a beneficial role in preventing cardiovascular disease in patients with gout. In addition, $\mathrm{LY} \%$ and NE\% may have a weak protective effect on the remodeling of heart structure and function in patients with gout, although the specific mechanism remains unclear. In the future, the sample size should be expanded to allow for more in-depth research.

\section{Declarations}

Funding information: This work was supported by the National Natural Science Foundation of China (No. 81603441), the Basic Application Research Project of Sichuan Science and Technology Department (No. 2017JY0297), Key Projects of Health Commission of Sichuan Province (No. 18ZD041), the Innovative Team Building Project of Chengdu Medical College (No. CYTD18-03), the School Cooperation Project of Nanchong (No. NSMC20170452), and the Key Projects of The First Affiliated Hospital of Chengdu Medical College (No. CYFY2019ZD01), Project of Sichuan Provincial Administration of traditional Chinese Medicine (No. 2020JC0023). Scientific research project of Sichuan Provincial Health Commission (key research project, No. 19ZD004).

Conflict of interest: No conflicts of interest

Authors' contributions $\square W D$ conceived and designed the work. WD and DL contributed to analyses of imaging data, prepared the figures, and provided technical support. JH, HL and XX contributed to data collection and interpretation of data. DL drafted the manuscript. WD, JZ and JL critically revised the manuscript for important intellectual content. All authors read and approved the final manuscript.

Compliance with ethical standards: All participants provided informed written consent. This study was approved by the Medical Ethics Committee of the Affiliated Hospital of Chengdu Medical College.

Acknowledgments[This study was made possible by the collaborative efforts of doctors, nurses, and administrators at the recruiting hospital. We thank everyone who contributed their time and expertise.

\section{References}

1. Merriman T (2015) An update on the genetic architecture of hyperuricemia and gout. Arthritis Res Ther 17(1):98. https://doi.org/10.1186/s13075-0150609-2

2. Gancheva R, Koundurdjiev A, Ivanova M, Kundurzhiev T, Kolarov Z (2019) Obesity, echocardiographic changes and framingham risk score in the spectrum of gout: A cross-sectional study. Arch Rheumatol 34(2):176-185. https://doi.org/10.5606/ArchRheumatol.2019.7062

3. Huang H, Huang B, Li Y et al (2014) Uric acid and risk of heart failure: A systematic review and meta-analysis. Eur J Heart Fail 16(1):15-24. https://doi.org/10.1093/eurjhf/hft132

4. Lin JC, Lin CL, Chen MC et al (2015) Gout, not hyperuricemia alone, impairs left ventricular diastolic function. Arthritis Res Ther 17:323. https://doi.org/10.1186/s13075-015-0842-8

5. Rotar Z, Praprotnik S, Tomšič M (2011) Clinical contrasts with the american college of rheumatology/european league against rheumatism provisional definitions of remission in rheumatoid arthritis for clinical trials: Comment on the article by felson et al. Arthritis Rheum 63(11):3642-3643. https://doi.org/10.1002/art.30552

6. Bozkurt B, Aguilar D, Deswal A et al (2016) Contributory risk and management of comorbidities of hypertension, obesity, diabetes mellitus, hyperlipidemia, and metabolic syndrome in chronic heart failure: A scientific statement from the american heart association. Circulation 134(23):e535-e578. https://doi.org/10.1161/CIR.0000000000000450

7. Gancheva RN, Kundurdjiev Al, Ivanova MG, Kundurzhiev TG, Rashkov RK, Kolarov ZG (2015) Ultrasonographic measurement of carotid artery resistive index and diastolic function of the heart in gout patients. Rheumatol Int 35(8):1369-1375. https://doi.org/10.1007/s00296-015-3280-7 
8. Liang J, Jiang Y, Huang Y et al (2020) The comparison of dyslipidemia and serum uric acid in patients with gout and asymptomatic hyperuricemia: A cross-sectional study. Lipids Health Dis 19(1):31. https://doi.org/10.1186/s12944-020-1197-y

9. Causevic A, Semiz S, Macic Dzankovic A et al (2010) Relevance of uric acid in progression of type 2 diabetes mellitus. Bosn J Basic Med Sci 10(1):54-59. https://doi.org/10.17305/bjbms.2010.2736

10. Cui Y, Bu H, Ma X, Zhao S, Li X, Lu S. The relation between serum uric acid and hba1c is dependent upon hyperinsulinemia in patients with newly diagnosed type 2 diabetes mellitus. J Diabetes Res 2016:7184123. https://doi.org/10.1155/2016/7184123

11. Pascual E, Sivera F, Andrés M (2018) Managing gout in the patient with renal impairment. Drugs Aging 35(4):263-273. https://doi.org/10.1007/s40266018-0517-7

12. Choe JY, Park SH, Kim SK (2010) Serum cystatin c is a potential endogenous marker for the estimation of renal function in male gout patients with renal impairment. J Korean Med Sci 25(1):42-48. https://doi.org/10.3346/jkms.2010.25.1.42

13. Harada M, Izawa A, Hidaka $\mathrm{H}$ et al (2017) Importance of cystatin $\mathrm{c}$ and uric acid levels in the association of cardiometabolic risk factors in japanese junior high school students. J Cardiol 69(1):222-227. https://doi.org/10.1016/j.jjcc.2016.03.013

14. Yang WX, Ma Y, Hou YL, Wang YB, You CG (2019) Prevalence of hyperuricemia and its correlation with serum lipids and blood glucose in physical examination population in 2015-2018: A retrospective study. Clin Lab 65(8). https://doi.org/10.7754/Clin.Lab.2019.190338

15. Saito Y, Nakayama T, Sugimoto K, Fujimoto Y, Kobayashi Y (2015) Relation of lipid content of coronary plaque to level of serum uric acid. Am J Cardiol 116(9):1346-1350. https://doi.org/10.1016/j.amjcard.2015.07.059

16. Jung JH, Song GG, Ji JD et al (2018) Metabolic syndrome: Prevalence and risk factors in korean gout patients. Korean J Intern Med 33(4):815-822. https://doi.org/10.3904/kjim.2016.062

17. Dudziak M, Rawicz-Zegrzda D, Jankowska M, Dębska-Ślizień A, Wołyniec Z, Rutkowski B (2016) Tissue doppler imaging and intima-media thickness as noninvasive methods of cardiovascular risk stratification in patients after kidney transplantation. Transplant Proc 48(5):1535-1538. https://doi.org/10.1016/j.transproceed.2015.12.132

18. Naghavi M, Yen AA, Lin AW (2016) Tanaka H, Kleis S. New indices of endothelial function measured by digital thermal monitoring of vascular reactivity: Data from 6084 patients registry. Int J Vasc Med 2016:1348028. https://doi.org/10.1155/2016/1348028

19. Jujo K, Minami Y, Haruki S et al (2017) Persistent high blood urea nitrogen level is associated with increased risk of cardiovascular events in patients with acute heart failure. ESC Heart Fail 4(4):545-553. https://doi.org/10.1002/ehf2.12188

20. Fang X, Pan C, Chen Y et al (2017) Assessment of subclinical left ventricular changes in essential hypertensive patients with hyperuricemia: A threedimensional speckle-tracking echocardiography study. Clin Exp Hypertens 39(1):93-99. https://doi.org/10.1080/10641963.2016.1210626

21. Aronow WS (2017) Hypertension and left ventricular hypertrophy. Ann Transl Med 5(15):310. https://doi.org/10.21037/atm.2017.06.14

22. Katholi RE, Couri DM (2011) Left ventricular hypertrophy: Major risk factor in patients with hypertension: Update and practical clinical applications. Int $J$ Hypertens 2011:495349. https://doi.org/10.4061/2011/495349

23. Turkes GF, Uysal S, Demir T et al (2021) Associations between bioavailable vitamin $\mathrm{d}$ and remnant cholesterol in patients with type 2 diabetes mellitus. Cureus 13(2):e13248. https://doi.org/10.7759/cureus.13248

24. Zhao Z, Hou C, Ye X, Cheng J (2020) Echocardiographic changes in newly diagnosed type 2 diabetes mellitus patients with and without hypertension. Med Sci Monit 26:e918972. https://doi.org/10.12659/MSM.918972

25. von Bibra H, Paulus WJ, St John Sutton M, Leclerque C, Schuster T, Schumm-Draeger PM (2015) Quantification of diastolic dysfunction via the age dependence of diastolic function - impact of insulin resistance with and without type 2 diabetes. Int J Cardiol 182:368-374.

https://doi.org/10.1016/j.ijcard.2014.12.005

26. Kozakova M, Morizzo C, Fraser AG, Palombo C (2017) Impact of glycemic control on aortic stiffness, left ventricular mass and diastolic longitudinal function in type 2 diabetes mellitus. Cardiovasc Diabetol 16(1):78. https://doi.org/10.1186/s12933-017-0557-z

27. Welles CC, Ku IA, Kwan DM, Whooley MA Schiller N, Turakhia M (2012) Left atrial function predicts heart failure hospitalization in subjects with preserved ejection fraction and coronary heart disease: Longitudinal data from the heart and soul study. J Am Coll Cardiol 59(7):673-680.

https://doi.org/10.1016/j.jacc.2011.11.012

28. Douglas PS (2003) The left atrium: A biomarker of chronic diastolic dysfunction and cardiovascular disease risk. J Am Coll Cardiol 2003;42(7):1206-7. https://doi.org/10.1016/s0735-1097(03)00956-2

29. Einwoegerer CF, Domingueti CP (2018) Association between increased levels of cystatin c and the development of cardiovascular events or mortality: A systematic review and meta-analysis. Arq Bras Cardiol 111(6):796-807. https://doi.org/10.5935/abc.20180171

30. Zhang QB, Zhu D, Wen Z et al (2019) High levels of serum uric acid, cystain c and lipids concentration and their clinical significance in primary gouty arthritis patients. Curr Rheumatol Rev 15(2):141-145. https://doi.org/10.2174/1573397114666180705095625

31. Liu J, Sukhova GK, Sun JS, Xu WH, Libby P, Shi GP (2004) Lysosomal cysteine proteases in atherosclerosis. Arterioscler Thromb Vasc Biol 24(8):13591366. https://doi.org/10.1161/01.ATV.0000134530.27208.41

32. Wang J, Duan Y, Sluijter JP, Xiao J (2019) Lymphocytic subsets play distinct roles in heart diseases. Theranostics 9(14):4030-4046. https://doi.org/10.7150/thno.33112. eCollection 2019

33. Yılmaz M, Korkmaz H, Bilen MN, Uku Ö, Kurtoğlu E (2016) Could neutrophil/lymphocyte ratio be an indicator of coronary artery disease, coronary artery ectasia and coronary slow flow? J Int Med Res 44(6):1443-1453. https://doi.org/10.1177/0300060516664637

34. Burns JA, Sanchez C, Beussink L et al (2018) Lack of association between anemia and intrinsic left ventricular diastolic function or cardiac mechanics in heart failure with preserved ejection fraction. Am J Cardiol 122(8):1359-1365. https://doi.org/10.1016/j.amjcard.2018.06.045

Page $6 / 10$ 
Tables

Table1. Baseline characteristics and echocardiographic parameters of gout patients

\begin{tabular}{|c|c|c|}
\hline Characteristics & $\mathrm{N}(\%)$ & mean $\pm S D$ \\
\hline Age『year】 & 227 & $45.71 \pm 15.33$ \\
\hline $\mathrm{BM} \otimes \mathrm{kg} / \mathrm{m}^{2} \square$ & 227 & $26.01 \pm 3.77$ \\
\hline Course of disease $\Downarrow$ year $\rrbracket$ & 227 & $4.70 \pm 5.53$ \\
\hline Hypertension & 43囚19\%囚 & \\
\hline Diabetes & $28 \bigotimes 12 \% \bigotimes$ & \\
\hline Tophi & $104 \llbracket 46 \% \rrbracket$ & \\
\hline \multicolumn{3}{|c|}{ echocardiographic parameter } \\
\hline GLS『\%凹 & 187 & $-17.53 \pm 2.31$ \\
\hline $\mathrm{GCS} \otimes \% \rrbracket$ & 187 & $-30.16 \pm 5.01$ \\
\hline LVEF $₫ \% \bigotimes$ & 227 & $62.96 \pm 4.9$ \\
\hline $\mathrm{E} / \mathrm{Em}$ & 227 & $10.25 \pm 3.16$ \\
\hline IVS『mm囚 & 227 & $9.61 \pm 1.38$ \\
\hline LVPW『mm® & 227 & $9.96 \pm 5.34$ \\
\hline LVEDD『mm $\rrbracket$ & 227 & $44.87 \pm 6.01$ \\
\hline LAVIIImL/怄 & 196 & $24.1 \pm 7.09$ \\
\hline
\end{tabular}

BMI: body mass index; GLS: global longitudinal strain; GCS: global circumferential strain; LVEF: left ventricular ejection fraction; E: diastolic peak early transmission flow velocity; Em: peak early diastolic mitral annulus velocity; IVS: interventricular septum; LVPW: left ventricular posterior wall; LVEDD: left ventricular end-diastolic diameter; LAVI: left atrial volume index.

\section{Table2. Correlation analysis of SUA and laboratory indexes}

\begin{tabular}{|c|c|c|}
\hline \multirow[t]{2}{*}{ Characteristics } & \multicolumn{2}{|l|}{ SUA } \\
\hline & $r$ & $P$ \\
\hline Age & -0.147 & 0.029 \\
\hline Course of disease & 0.141 & 0.036 \\
\hline HGB & 0.149 & 0.028 \\
\hline CREA & 0.271 & $\otimes 0.001$ \\
\hline Cys-C & 0.165 & 0.015 \\
\hline TP & 0.195 & 0.004 \\
\hline TG & 0.242 & 0.001 \\
\hline TC & 0.162 & 0.023 \\
\hline ApoB & 0.155 & 0.031 \\
\hline FPG & -0.219 & 0.003 \\
\hline
\end{tabular}

Table3. Correlation analysis of echocardiographic parameters and laboratory indexes 


\begin{tabular}{|c|c|c|c|c|c|c|c|c|c|c|c|c|c|c|c|}
\hline Characteristics & GLS & & GCS & & LVEF & & $\mathrm{E} / \mathrm{Em}$ & & IVS & & LVPW & & LVEDD & & LAVI \\
\hline & $r$ & $P$ & $r$ & $P$ & $r$ & $P$ & $r$ & $P$ & $r$ & $P$ & $r$ & $P$ & $r$ & $P$ & $r$ \\
\hline Age & 0.119 & 0.106 & 0.038 & 0.601 & 0.028 & 0.676 & 0.503 & $\begin{array}{l}\square \\
0.001\end{array}$ & 0.318 & $\begin{array}{l}0.001 \\
0.01\end{array}$ & 0.258 & $\begin{array}{l}0.001 \\
0.01\end{array}$ & 0.068 & 0.307 & 0.403 \\
\hline BMI & 0.179 & 0.014 & 0.022 & 0.762 & -0.041 & 0.538 & 0.183 & 0.006 & 0.335 & 0.001 & 0.335 & 0.001 & 0.234 & $\begin{array}{l}0.001 \\
0\end{array}$ & 0.049 \\
\hline $\begin{array}{l}\text { Course of } \\
\text { disease }\end{array}$ & 0.246 & 0.001 & 0.125 & 0.088 & -0.028 & 0.671 & 0.221 & 0.001 & 0.280 & 0.001 & 0.294 & 0.001 & 0.066 & 0.324 & 0.173 \\
\hline WBC & 0.098 & 0.19 & 0.006 & 0.937 & 0.015 & 0.828 & 0.051 & 0.448 & 0.135 & 0.046 & 0.158 & 0.019 & 0.067 & 0.326 & 0.003 \\
\hline RBC & 0.032 & 0.667 & -0.06 & 0.418 & 0.013 & 0.85 & -0.324 & 0.001 & -0.192 & 0.004 & -0.108 & 0.111 & -0.072 & 0.291 & -0.325 \\
\hline HGB & -0.007 & 0.929 & -0.104 & 0.161 & -0.038 & 0.58 & -0.419 & $\begin{array}{l}0.001 \\
0\end{array}$ & -0.261 & $\begin{array}{l}0.001 \\
0.01\end{array}$ & -0.187 & 0.005 & -0.106 & 0.119 & -0.347 \\
\hline PLT & -0.032 & 0.666 & -0.014 & 0.856 & 0.041 & 0.547 & -0.190 & 0.005 & -0.059 & 0.384 & 0.042 & 0.534 & 0.162 & 0.016 & -0.123 \\
\hline NE\% & 0.026 & 0.732 & 0.133 & 0.073 & 0.021 & 0.754 & 0.264 & $\begin{array}{l}0.001 \\
0\end{array}$ & 0.200 & 0.003 & 0.124 & 0.067 & 0.04 & 0.558 & 0.074 \\
\hline LY\% & -0.023 & 0.763 & -0.189 & 0.011 & -0.026 & 0.707 & -0.302 & 0.001 & -0.243 & 0.001 & -0.142 & 0.036 & -0.077 & 0.256 & -0.103 \\
\hline UREA & 0.212 & 0.004 & 0.211 & 0.004 & -0.036 & 0.591 & 0.262 & 0.001 & 0.357 & $\begin{array}{l}0.001 \\
0\end{array}$ & 0.275 & $\begin{array}{l}0.001 \\
0.01\end{array}$ & 0.055 & 0.412 & 0.214 \\
\hline CREA & -0.015 & 0.842 & 0.019 & 0.803 & -0.043 & 0.524 & 0.094 & 0.162 & 0.200 & 0.003 & 0.144 & 0.032 & 0.032 & 0.641 & 0.187 \\
\hline Cys-C & 0.190 & 0.01 & 0.14 & 0.061 & -0.028 & 0.676 & 0.301 & $\begin{array}{l}0.001 \\
0.01\end{array}$ & 0.372 & 0.001 & 0.323 & $\begin{array}{l}0.001 \\
0\end{array}$ & 0.037 & 0.587 & 0.304 \\
\hline$\beta 2-M G$ & 0.159 & 0.035 & 0.150 & 0.047 & 0.006 & 0.935 & 0.325 & 0.001 & 0.343 & 0.001 & 0.255 & 0.001 & 0.095 & 0.168 & 0.344 \\
\hline TP & 0.017 & 0.824 & -0.015 & 0.838 & -0.089 & 0.19 & -0.198 & 0.003 & -0.211 & 0.002 & -0.172 & 0.011 & -0.102 & 0.131 & -0.06 \\
\hline ALB & 0.014 & 0.847 & -0.005 & 0.944 & -0.129 & 0.057 & -0.317 & 0.001 & -0.200 & 0.003 & -0.252 & $\begin{array}{l}0.001 \\
0.01\end{array}$ & -0.263 & ${ }^{0} .001$ & -0.227 \\
\hline $\mathrm{A} / \mathrm{G}$ & -0.009 & 0.902 & 0.005 & 0.944 & -0.05 & 0.46 & -0.163 & 0.015 & -0.088 & 0.195 & -0.173 & 0.01 & -0.212 & 0.002 & -0.216 \\
\hline TG & 0.175 & 0.026 & -0.026 & 0.743 & -0.101 & 0.159 & 0.073 & 0.306 & 0.170 & 0.017 & 0.165 & 0.021 & -0.05 & 0.486 & -0.094 \\
\hline TC & 0.13 & 0.1 & 0.137 & 0.083 & -0.153 & 0.032 & -0.059 & 0.412 & 0.019 & 0.788 & -0.014 & 0.85 & -0.131 & 0.066 & -0.177 \\
\hline HDL-C & -0.112 & 0.159 & -0.019 & 0.808 & 0.008 & 0.913 & -0.093 & 0.193 & -0.138 & 0.053 & -0.189 & 0.008 & -0.101 & 0.156 & -0.01 \\
\hline LDL-C & 0.148 & 0.062 & 0.148 & 0.061 & -0.160 & 0.025 & -0.085 & 0.233 & 0.033 & 0.641 & 0.016 & 0.824 & -0.105 & 0.144 & -0.212 \\
\hline ApoA-I & -0.129 & 0.104 & -0.058 & 0.471 & 0.057 & 0.428 & -0.039 & 0.584 & -0.075 & 0.296 & -0.136 & 0.058 & -0.212 & 0.003 & -0.082 \\
\hline ApoB & 0.125 & 0.115 & 0.095 & 0.235 & -0.150 & 0.037 & -0.044 & 0.539 & 0.018 & 0.807 & 0.002 & 0.982 & -0.108 & 0.133 & -0.144 \\
\hline ApoA-I/ApoB & -0.175 & 0.028 & -0.078 & 0.329 & 0.168 & 0.019 & -0.017 & 0.809 & -0.061 & 0.396 & -0.088 & 0.224 & -0.054 & 0.453 & 0.048 \\
\hline Hcy & 0.058 & 0.476 & 0.118 & 0.145 & 0.037 & 0.613 & 0.12 & 0.099 & 0.169 & 0.02 & 0.193 & 0.008 & 0.117 & 0.108 & 0.132 \\
\hline $\mathrm{Lp}(\mathrm{a})$ & -0.074 & 0.363 & -0.023 & 0.776 & 0.115 & 0.116 & 0.162 & 0.026 & 0.019 & 0.792 & 0.051 & 0.485 & 0.079 & 0.282 & 0.167 \\
\hline FPG & 0.164 & 0.044 & 0.068 & 0.409 & -0.208 & 0.005 & 0.055 & 0.458 & 0.054 & 0.467 & 0.032 & 0.665 & -0.039 & 0.605 & 0.04 \\
\hline HBA1C & 0.262 & 0.002 & 0.093 & 0.287 & -0.073 & 0.353 & 0.136 & 0.085 & 0.164 & 0.037 & 0.246 & 0.002 & 0.156 & 0.047 & 0.173 \\
\hline$A G$ & 0.241 & 0.004 & 0.111 & 0.2 & -0.07 & 0.377 & 0.125 & 0.111 & 0.169 & 0.031 & 0.235 & 0.003 & 0.169 & 0.031 & 0.168 \\
\hline CRP & 0.113 & 0.142 & 0.167 & 0.03 & 0.041 & 0.564 & 0.184 & 0.008 & 0.170 & 0.015 & 0.164 & 0.019 & 0.279 & $\begin{array}{l}0.001 \\
0\end{array}$ & 0.123 \\
\hline ESR & 0.019 & 0.813 & 0.189 & 0.017 & 0.037 & 0.608 & 0.306 & $\begin{array}{l}0.001 \\
0\end{array}$ & 0.218 & 0.002 & 0.179 & 0.012 & 0.172 & 0.016 & 0.211 \\
\hline
\end{tabular}

BMI: body mass index; WBC: white blood cell; RBC: red blood cell; HGB: hemoglobin; PLT: platelets; NE\%: the percentage of neutrophils; LY\%: the percentage o

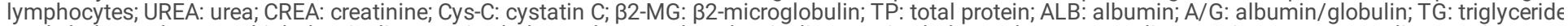
total cholesterol; HDL-C: high density lipoprotein cholesterol; LDL-C: low-density lipoprotein cholesterol; ApoA-l: apolipoprotein A-l; ApoB: apolipoprotein B; Hcy homocysteine; Lp(a): lipoprotein(a); FPG: fasting plasma glucose; HBA1C: glycated hemoglobin; AG: average blood glucose; CRP: c-reactive protein; ESR: eryt sedimentation rate. 

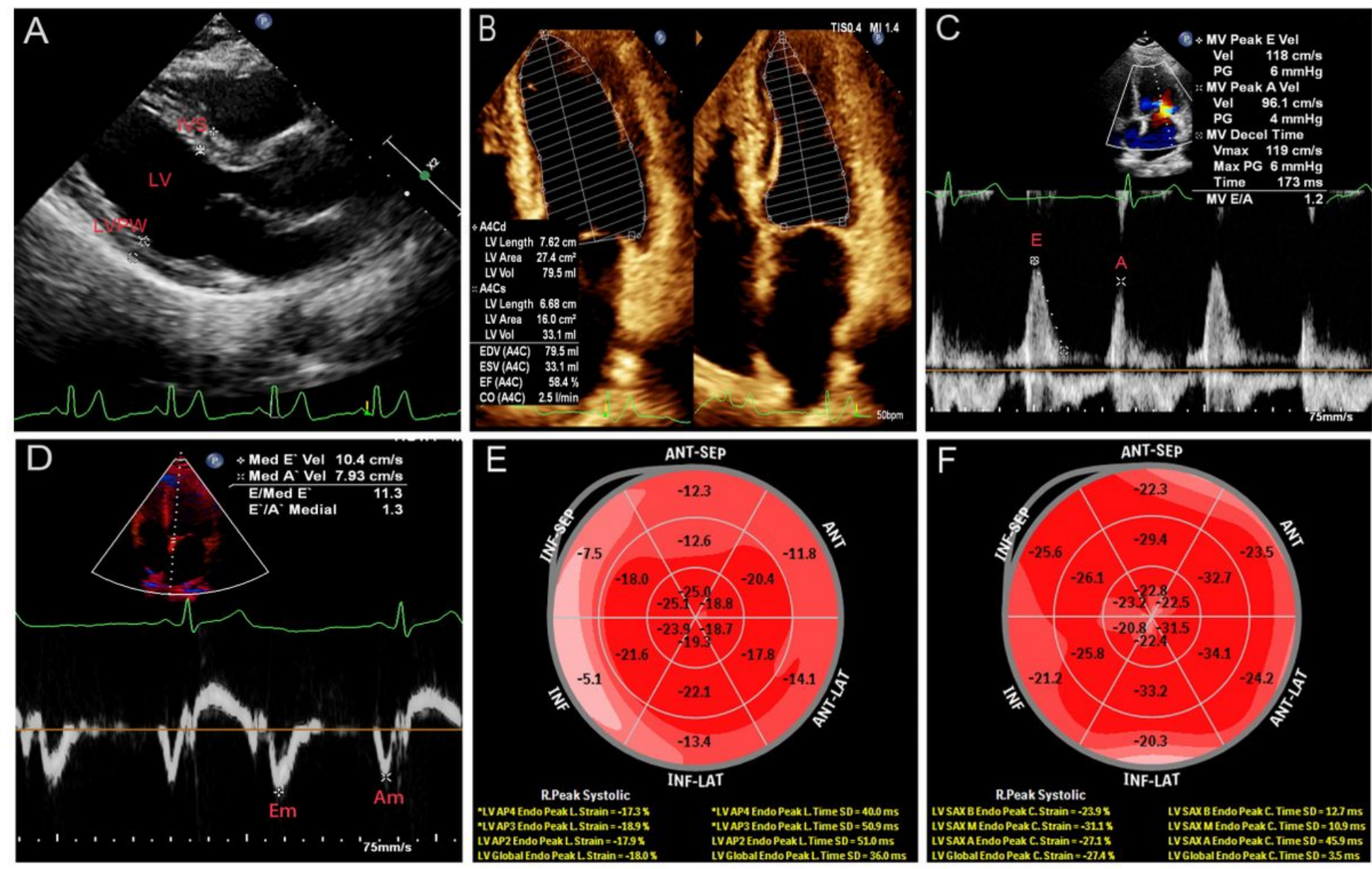

Figure 1

Echocardiographic examination of the heart in patients with gout Figure1-A. IVS, LVPW, and LVEDD of patients with gout were measured by transthoracic echocardiography on long axis section of left ventricle; Figure1-B.LVEF was calculated using the Simpson method; Figure1-C.Pulsed wave doppler was used to measure the $\mathrm{E}$ peak of mitral orifice flow spectrum in patients with gout;Figure1-D.Tissue doppler imaging was used to measure Em peaks at the mitral annulus septum in patients with gout; Figure1-E. GLS in patients with gout was measured by two-dimensional speckle tracing imaging; Figure1-F. GCS in patients with gout was measured by two-dimensional speckle tracing imaging.

A

GCS $\geqslant-29.9 \%$ vs GCS $<-29.9 \%$
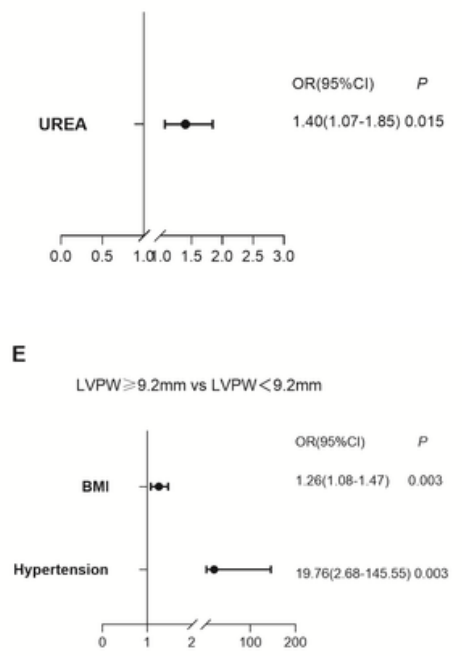

B LVEF $\leqslant 62.7 \%$ vs LVEF $>62.7 \%$

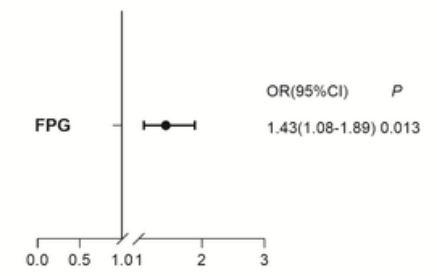

$\mathbf{F}$

LVEDD $\geqslant 45.6 \mathrm{~mm}$ vs LVEDD $<45.6 \mathrm{~mm}$

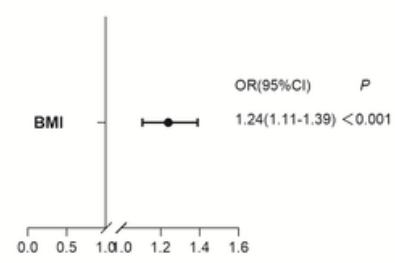

c

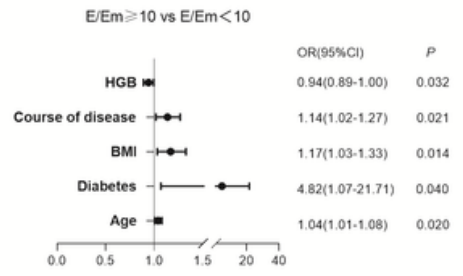

G

LAVI $\geqslant 23.2 \mathrm{~mL} / \mathrm{m}^{\prime}$ vs $L A V I<23.2 \mathrm{~mL} / \mathrm{m}$
D

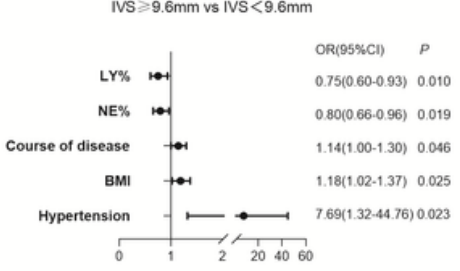

Figure 2 
Logistics regression analysis of echocardiographic parameters and laboratory indicators in patients with gout Figure 2-A: UREA was the risk factor for absolute reduction in GCS [OR $(95 \% \mathrm{Cl})=1.40(1.07-1.85), \mathrm{p}=0.015]$; Figure 2-B: FPG was a risk factor for LVEF reduction [OR $(95 \% \mathrm{Cl})=1.43(1.08-1.89)$, $\mathrm{P}$ $=0.013]$; Figure 2-C: Age, BMI, course of disease and diabetes were the risk factors for increasing $\mathrm{E} / \mathrm{Em}[\mathrm{OR}(95 \% \mathrm{Cl})=1.04(1.01-1.08), 1.17(1.03-1.33), 1.14$ (1.02-1.27), 4.82 (1.07-21.71), $\mathrm{p}=0.020,0.014,0.021,0.040$, respectively.], $\mathrm{HGB}$ may reduce the increased risk of $\mathrm{E} / \mathrm{Em} \mathrm{[OR}(95 \% \mathrm{Cl})=0.94(0.89-1.00), \mathrm{p}$ $=0.032$ ]; Figure 2-D: BMI, course of disease, and hypertension were the risk factors for IVS thickening [OR $(95 \% \mathrm{Cl})=1.18(1.02-1.37), 1.14(1.00-1.30), 7.69$ (1.32-44.76), $\mathrm{p}=0.025,0.046,0.023$, respectively.], NE\% and LY\% may reduce the risk of IVS thickening [OR $(95 \% \mathrm{Cl})=0.80(0.66-0.96), 0.75(0.60-0.93), p$ $=0.019,0.010$, respectively]. Figure 2-E: BMI and hypertension were risk factors for LVPW thickening [OR $(95 \% \mathrm{Cl})=1.26(1.08-1.47), 19.76(2.68-145.55), \mathrm{p}$ $=0.03,0.03$, respectively.]; Figure 2-F: BMI was a risk factor for increased LVEDD [OR (95\%Cl) =1.24 (1.11-1.39), p < 0.001]; Figure 2-G: ALB, Cys-C and course of disease were the increased risk factors for LAVI [OR $(95 \% \mathrm{Cl})=1.20(1.01-1.43), 105.47(1.18-9468.03), 1.14(1.01-1.28), p=0.035,0.042,0.028$, respectively.] 